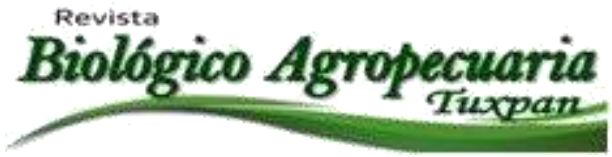

\title{
Identificación de la diversidad faunística y florística de tres diferentes zonas del Estado de Veracruz. Percepción de la comunidad ganadera
}

Identification of the faunistic and floristic diversity of three different zones of Veracruz State.

Perception livestock's community

\author{
Castro-José Carlos Nahin ${ }^{1}$, Ana Lid Del Angel-Pérez ${ }^{1}$ y Mabel Hernández-Osorio ${ }^{1}$ \\ ${ }^{1}$ Instituto Nacional de Investigaciones Forestales Agrícolas y Pecuarias (INIFAP) Km 34.5 Carr. \\ Veracruz-Córdoba. 94277. Medellín de Bravo, Veracruz. \\ ${ }^{凶}$ Autor para correspondencia: nahin.castro@gmail.com
}

Recibido: 07/01/2014

Aceptado: 21/07/2014

\section{RESUMEN}

Para el 2011 el estado de Veracruz contaba con 3,963,610 cabezas de ganado, posicionándose como el principal productor de ganado bovino del país, una ganadería que se desarrolla principalmente bajo el sistema extensivo y que al ir necesitando mayores áreas de pastoreo contribuye significativamente al cambio de uso de suelo y a la vez, cambios de los ecosistemas en donde se desarrolla, entre los que destacan las modificaciones de la estructura de la flora y fauna. Basados en esto y considerando al sector ganadero parte fundamental para la toma de decisiones en cuanto a actividades de conservación se refiere se realizaron 200 encuestas tipo referéndum en la zona Norte, Centro y Sur del estado de Veracruz, abarcando un total de doce municipios con antecedentes de actividades ganaderas. La encuesta solicitaba entre otras cosas, información acerca de la flora y fauna que los propietarios tienen y han visto en sus terrenos, las respuestas se organizaron en matrices y se sometieron a un análisis simple. La fauna se agrupo por clase taxonómica (Mammalia, Aves, Sauropsida) siendo las aves la clase con mayor porcentaje de registros, $53.94,44.95$ y $38.53 \%$ para cada una de la zonas. La flora se agrupo de acuerdo al uso que se le da, (forraje, consumo humano, maderable) la vegetación utilizada para consumo humano tuvo el mayor porcentaje de registros para las tres zonas con valores de 42.55, 34.82 y $42 \%$ respectivamente. Es importante considerar que los encuestados no mencionaron a los insectos y otros invertebrados de menor tamaño.

Palabras claves: ganadería, biodiversidad, invertebrados, conservación, suelos.

Revista Científica Biológico Agropecuaria Tuxpan 2 (1)

ISSN: 2007-6940 


\begin{abstract}
In 2011 the state of Veracruz had 3,963,610 cattle, positioning itself as the leading producer of cattle in the country, a herd that develops mainly under the extensive system and that going to require increased grazing areas contributes significantly to the change of use while soil, ecosystem changes where it develops, among which changes in the structure of flora and fauna. Based on this and considering the livestock sector essential part for making decisions about conservation activities referred 200 type surveys were conducted referendum in the north, center and south of the state of Veracruz, encompassing a total of twelve municipalities with history livestock activities. The survey asked, among other things, information about the flora and fauna that owners have and have seen their land; the responses were organized into matrices and subjected to a simple analysis. The fauna was grouped by taxonomic class (Mammalia, Birds, and Sauropsida) birds being the class with the highest percentage of records, 53.94, 44.95 and $38.53 \%$ for each of the areas. The flora was grouped according to the use that is given, (fodder, human consumption, timber) vegetation used for human consumption had the highest percentage of records for the three areas with values of $42.55,34.82$ and $42 \%$ respectively. It is important to consider that the respondents did not mention insects and other smaller invertebrates.
\end{abstract}

Keywords: livestock, biodiversity, invertebrates, conservation, soil.

\section{INTRODUCCIÓN}

El uso y el cambio de uso de la tierra relacionados con la producción ganadera modifican ecosistemas que son los hábitats de determinadas especies (FAO, 2009.) Los animales pastando se asocian frecuentemente con sobrepastoreo, degradación del suelo y deforestación (FAO, 1999) así como con una ampliación de la frontera agrícola en busca de tierras más fértiles, y en consecuencia una contribución a la reducción de los recursos naturales (Loening et al. 2003), esto implica una pérdida de diversidad de flora y de fauna, y una reducción de la capacidad del ecosistema de proporcionar servicios ambientales. En el estado de Veracruz, la ganadería ocupa cerca del $69 \%$ de la tierra laborable, esta áreas se consideran como deforestadas debido a que están bajo el monocultivo de pastos (Landeros., 2010). La introducción de este tipo de especies extranjeras ejercen impactos negativos en la composición de especies, estructura y funcionamiento de los ecosistemas invadidos (Peralta, 2007) En México los estudios sobre cuestiones ambientales basados en la percepción de los habitantes de las zonas rurales señalan a estos como los principales usuarios y propietarios de los ambientes naturales y se les considera como parte fundamental en la toma de decisiones para la conservación del ecosistema, así pues, dentro de las zonas ganaderas, es la población la que puede brindar información fiable acerca de su estado en determinado espacio-tiempo. (Castillo et al. 2009 y Guevara et al. 2011). Por ello el objetivo principal del presente trabajo fue determinar que especies de flora y fauna son reconocidas en las regiones ganaderas del estado de Veracruz, considerando la percepción de las personas pertenecientes a dichas regiones.

\section{MATERIALES Y METODOS}

Se aplicaron 200 encuestas tipo referéndum a la comunidad ganadera de 12 municipios del estado de Veracruz abarcando tres zonas de estudio, Norte (40 encuestas), Centro (85 encuestas) y Sur (75 encuestas). Los

\title{
Revista Científica Biológico Agropecuaria Tuxpan 2 (1) ISSN: 2007-6940
}


municipios fueron Acayucan, Cd. Isla, La Antigua, Medellín, Nautla, Palma Sola, Paso de Ovejas, San Andrés Tuxtla, San Rafael, Santiago Tuxtla, Vega de Alatorre y Veracruz. Se les cuestiono entre otras cosas, cual es el tipo de fauna que han visto dentro de los terrenos destinados a actividades de ganadería extensiva así como la flora que se encuentra dentro de los mismos. Para analizar la fauna se establecieron tres diferente grupos taxonómicos (Mammalia, aves y Sauropsida); los resultados se organizaron en matrices y se les realizo un análisis simple registrándose los nombres de las especies animales y el uso de las especies vegetales, los valores obtenidos fueron analizados respecto al número total de registros, en el caso de la fauna se determinó a que familia pertenecían y para las especies vegetales la especie.

\section{RESULTADOS Y DISCUSION}

\section{Percepción de la diversidad faunística por parte de la comunidad ganadera}

Los resultados mostraron que el grupo de las aves fue el que obtuvo el mayor número de registros, así como el porcentaje más alto respecto al total de registros en las tres zonas (Cuadro 1). De la misma forma se obtuvo un total de 8 familias identificadas por los productores ganaderos para la zona norte y 10 para la zona centro y sur. Es importante mencionar que para el caso de las aves el número de especies en la región ganadera está muy por debajo de lo que muestran otros estudios en donde se registraron un total de 99 especies en pastizales ganaderos de Veracruz, (Bojorges y López, 2006)., ante esta situación se debe considerar que los encuestados tienen poco o nulo conocimiento acerca de la diversidad de especies que habitan en sus terrenos así como si estas son residentes o migratorias, esto pudo haber contribuido de manera significativa a que hallan mencionado una baja cantidad de especies de avifauna. $\mathrm{Si}$ bien, el número de aves a pesar de ser inferior a lo reportado por el autor, sugiere que existen condiciones que favorecen la presencia de estas, y hay quienes afirman que en las áreas de ganadería extensiva donde se utilizan cercos vivos junto con el pastizal; generalmente aún conservan parches de vegetación nativa que funcionan como zonas de alimentación y anidamiento que favorecen la presencia de estos organismos, (Zapata et al. 2007, Murgueitio y Calle, 1998) así pues, es muy probable que las aves encuentren una fuente de alimento adecuado, particularmente insectos, los cuales también encuentran refugio en esta zonas y que sin embargo no fueron mencionados por los entrevistados debido al desconocimiento de los mismos. La zona centro presenta el mayor número y porcentaje de registro de mamíferos con un total de 6 especies. De manera general la clase Sauropsida presento los valores más bajos en comparación con los otros grupos taxonómicos, así como un número menor de especies con un total de 4, es interesante observar para esta clase que la zona Sur es la que presento el mayor registro de las tres zonas estudiadas. Los primeros conquistadores españoles reportaron frondosos bosques de galería, con grandes árboles en los que la vida silvestre nativa de estos ambientes primarios era rica en variedad, (Del Angel et al. 2006) el paisaje actual dista mucho de aquellos parajes que existían antes de la introducción de la actividad ganadera, en la actualidad Veracruz es uno de los estados con mayor diversidad de ecosistemas, es también el principal productor ganadero bajo el sistema extensivo, el cual ha llevado a extender los territorios de pastura al punto de modificar de forma total la estructura del paisaje, estas modificaciones comprometen el hábitat de animales terrestres que a diferencia de las aves, utilizan el ecosistema como zonas de refugio, alimentación y reproducción de forma más directa, los organismos al necesitar áreas para reproducirse $\mathrm{o}$ alimentarse, se desplazan en busca de lugares idóneos, muchos de ellos con hábitos nocturnos, por lo que es

Revista Científica Biológico Agropecuaria Tuxpan 2 (1)

ISSN: 2007-6940 
más difícil tener contacto visual con ellos y puede ser la razón por la cual los registros obtenidos para las clases taxonómicas
Mammalia y Sauropsida muestran valores más bajos del total de registros en las tres zonas (Cuadro 1).

Cuadro 1. Porcentaje de registros por grupo taxonómico respecto al total de fauna registrada en cada una de las zonas de estudio.

\begin{tabular}{c|cccccc}
\hline \multicolumn{1}{c}{ AREA DE ESTUDIO } \\
\hline $\begin{array}{c}\text { CLASE } \\
\text { TAXONOMICA }\end{array}$ & \multicolumn{2}{c}{ ZONA NORTE } & \multicolumn{2}{c}{ ZONA CENTRO } \\
\cline { 2 - 7 } & $\begin{array}{c}\text { Numero } \\
\text { de } \\
\text { registros }\end{array}$ & $\begin{array}{c}\text { \% de registros } \\
\text { (TR* }=152)\end{array}$ & $\begin{array}{c}\text { Número de } \\
\text { registros }\end{array}$ & $\begin{array}{c}\text { \% de registros } \\
\text { (TR=347) }\end{array}$ & $\begin{array}{c}\text { Número de } \\
\text { registros }\end{array}$ & \% de registros (TR=327) \\
\hline Mammalia & 43 & 28.28 & 142 & 40.92 & 126 & 38.53 \\
Aves & 82 & 53.94 & 156 & 44.95 & 126 & 38.53 \\
Sauropsida & 27 & 17.76 & 49 & 14.12 & 75 & 22.93 \\
\hline
\end{tabular}

* $\mathrm{TR}=$ total de registros de fauna.

Al no haber una descripción exacta por parte de los entrevistados, los animales mencionados se clasificaron por familias taxonómicas en el Cuadro 2, se muestran las familias más representativas en cada una de las zonas y el total de menciones para cada una. El grupo de los insectos no está dentro de las percepciones que tienen la comunidad ganadera dentro de sus terrenos. Al ser un grupo con organismos de pequeño tamaño, no son considerados como importantes, al respecto, los productores ganaderos desconocen los beneficios que pueden aportar este tipo de fauna al ecosistema; haciendo referencia específica a los escarabajos estercoleros, los cuales se pueden ver afectados por actividades como la aplicación de antiparasitarios y herbicidas, sin embargo dichas actividades no solo afectan a los escarabajos, sino a toda la fauna edáfica en general (Martínez y Cruz, 2009).

Cuadro 2. Familias faunísticas más representativas en las zonas con actividades ganaderas.

\begin{tabular}{|c|c|c|c|c|c|c|c|c|}
\hline \multicolumn{4}{|c|}{ ZONA NORTE } & \multicolumn{2}{|c|}{ ZONA CENTRO } & \multicolumn{3}{|c|}{ ZONA SUR } \\
\hline Mammalia & Aves & Sauropsida & Mammalia & Aves & Sauropsida & Mammalia & Aves & Sauropsida \\
\hline $\begin{array}{l}\text { Leporidae } \\
\text { (14) }\end{array}$ & $\begin{array}{l}\text { Icteridae } \\
\text { (13) }\end{array}$ & $\begin{array}{c}\text { Colubridse } \\
\text { (9) }\end{array}$ & $\begin{array}{c}\text { Canidae } \\
(40)\end{array}$ & $\begin{array}{l}\text { Tyrannidee } \\
\text { (26) }\end{array}$ & $\begin{array}{l}\text { Colubridse } \\
\text { (22) }\end{array}$ & $\begin{array}{c}\text { Leporidae } \\
\text { (42) }\end{array}$ & $\begin{array}{l}\text { Crscidae } \\
\text { (20) }\end{array}$ & $\begin{array}{c}\text { Colubridge } \\
\text { (32) }\end{array}$ \\
\hline & $\begin{array}{c}\text { Tyrannidse } \\
\text { (13) }\end{array}$ & & & & & & & \\
\hline
\end{tabular}

\section{Percepción de la diversidad florística por parte de la comunidad ganadera}

Para el caso de las especies vegetales que se encuentran en las zonas ganaderas, las especies que son destinadas para el consumo humano registraron el mayor porcentaje de registros para las tres zonas de estudio (Cuadro 3), de las especies mencionadas Mangifera indica fue la que obtuvo mayor registro en todas, esto muestra que las áreas ganaderas no presentan exclusividad en cuanto al uso que se

\section{Revista Científica Biológico Agropecuaria Tuxpan 2 (1) ISSN: 2007-6940}


le da, ya que además de los pastos utilizados como forraje las personas tienden a establecer especies que pueden ser aprovechadas para el autoconsumo, estas especies pueden encontrarse ya sea al interior de los terrenos o establecidas en la periferia funcionando como cercos vivos y proveedores de frutos ( Zapata et al. 2007).

Cuadro 3. Número de registros para las especies vegetales identificadas por la comunidad ganadera.

\begin{tabular}{c|cccccc}
\hline \multicolumn{7}{c}{ AREA DE ESTUDIO } \\
\hline $\begin{array}{c}\text { USOS DE LA } \\
\text { FLORA }\end{array}$ & \begin{tabular}{c} 
ZONA NORTE \\
\cline { 2 - 7 } \\
$\begin{array}{c}\text { Numero } \\
\text { de de registros } \\
\text { registro } \\
\text { (TR }=329)\end{array}$
\end{tabular} & $\begin{array}{c}\text { Numero de } \\
\text { registros }\end{array}$ & $\begin{array}{c}\text { ZONA de registros } \\
\text { (TR=962) }\end{array}$ & $\begin{array}{c}\text { Numero de } \\
\text { registros }\end{array}$ \\
\hline Forraje & 84 & 25.53 & 301 & 31.28 & 169 & 28.74 \\
Consumo de registros (TR=588) \\
Maderables & 105 & 31.91 & 335 & 34.82 & 247 & 42.00 \\
\hline
\end{tabular}

$* \mathrm{TR}=$ total de registros de flora.

Las especies maderables ocupan el segundo sitio en cuanto a porcentaje de registros siendo Cedrela odorata la especie que más se mencionó, (cuadro 4), los resultados muestran que los ganaderos conocen el valor del cedro rojo y por ello lo consideran parte importante de sus terrenos, el cedro rojo (Cedrela odorata L.) junto con la caoba (Swietenia macrophylla King) son conocidas como dos de las especies preciosas más importantes para el trópico mexicano (Ramírez et al,. 2008) Finalmente las especies forrajeras obtuvieron el menor porcentaje de registros para las tres zonas; para la zona Norte y Centro Bursera simaruba fue la que obtuvo mayores registros y para la zona Sur Gliricidia sepium fue la especie principal, si bien ambas especies han sido ampliamente utilizadas como complemento nutricional del ganado bovino, para el caso de Gliricidia sepium existe poca disponibilidad por parte de los habitantes cercanos a la costa para producirla ya que consideran que la naturaleza las produce sin la necesidad de cultivarlas (Moreno et al. 2011 ) Aunque estas especies son consideradas forrajeras, no se producen de manera constante y se encuentran formando parte de los cercos vivos de los terrenos, siendo la reproducción a partir de esquejes la forma más común de multiplicarla. Así pues, bajo estas condiciones, las especies consideradas forrajeras en las zonas de estudio serian un complemento, más no un sustituto de la alimentación proporcionada por los pastos, aunque hay que mencionar que la utilización de sistemas agrosilvopastoriles en los que se manejen especies forrajeras como Leucaena leucocephala, Gliricidia sepium entre otras, más allá de los cercos vivos podría contribuir de manera significativa a incrementar los rendimientos y reducir la áreas degradadas. (Olivares et al. 2011; Zapata et al. 2007).

Cuadro 4. Especie de flora más representativa en áreas de ganadería extensiva. 


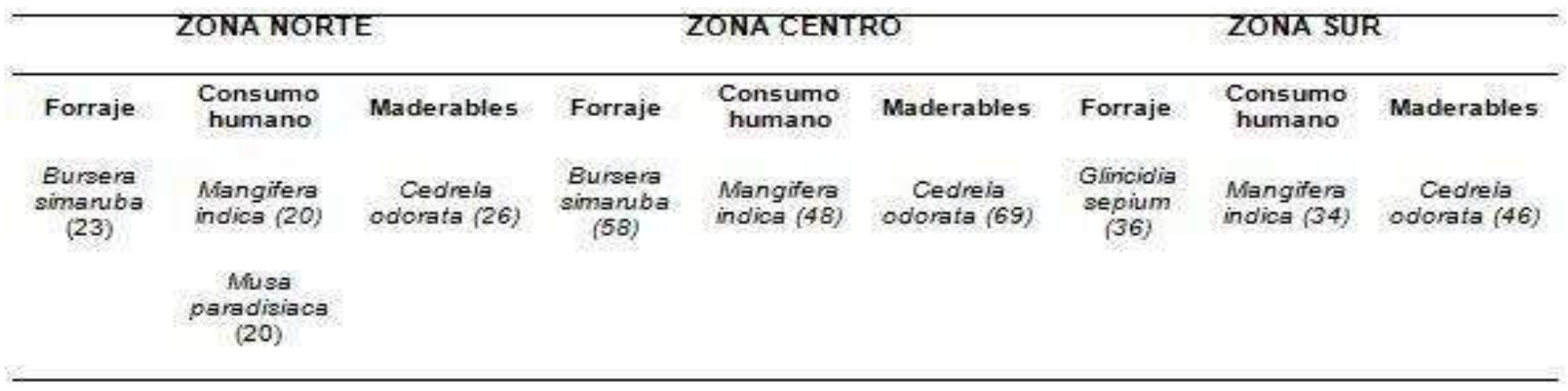

\section{CONCLUSIONES}

Los ganaderos tienen conocimiento sobre las especies de mayor tamaño que se encuentran dentro de sus terrenos, algunas de ellas no son residentes permanentes debido principalmente a la falta de áreas de refugio y anidamiento para el caso de las aves, por otra parte, las áreas ganaderas por sus características permiten el tránsito en busca de espacios para el desarrollo, reproducción y alimentación de la fauna, por lo que de alguna manera contribuyen a mantener conectados los remanentes de bosque perturbado o acahuales. Los organismos de tamaño menor, como los insectos y demás invertebrados no figuraron entre las respuestas de los entrevistados, esto pudo deberse en gran parte primero, por el tamaño, y segundo porque no obtienen un beneficio directo de ellos, de alguna manera para un productor son especies no deseadas en sus pastizales, para esto, una mejora en la difusión de los beneficios que obtiene el ecosistema a través de estos organismos puede contribuir a aumentar el conocimiento local y poder mejorar las condiciones ambientales en las que se desarrollan. En el caso de la flora, los frutales fueron los más mencionados, aunque no se encuentran establecidos como un cultivo, forman parte de los paisajes ganaderos como proveedores de alimento para los habitantes de la zona, de manera general, especies consideradas dentro del estrato herbáceo no fueron mencionadas, siendo especies del tipo arborescente las que predominaron en las respuesta de los entrevistados, esto puede deberse, a que el monocultivo con pastos forrajeros limita el desarrollo de especies nativas en este estrato, disminuyendo así la diversidad florística.

\section{LITERATURA CITADA}

Castillo, A., Corral, V., González, E., Paré, L., Paz, M., Reyes, J. y Schteingart, M.. 2009. Conservación y sociedad, en Capital Natural de México, Vol. II: Estado de conservación y tendencias de cambio. CONABIO, México, 761-801 p.

Cruz, J. y López, L. 2006. Asociación de la riqueza y diversidad de especies de aves y estructura de la vegetación en una selva mediana subperennifolia en el centro de Veracruz, México. Revista Mexicana de Biodiversidad. 77: 235249.

https://doi.org/10.22201/ib.20078706e.2006.002.3 38

Del Angel, A., Villagómez, J., Mendoza, M., Rebolledo, A. 2006. Valoración de recursos naturales y ganadería en la zona centro del Veracruz, México. Maderas y bosques. 12 (2): 29-48. https://doi.org/10.21829/myb.2006.1221241

FAO (Organización de las Naciones Unidas para la Agricultura y la Alimentación) 2009. El estado actual de la agricultura y la alimentación. La ganadería a examen. Roma, Italia. 200 p. https://doi.org/10.18356/074122cc-es

\section{Revista Científica Biológico Agropecuaria Tuxpan 2 (1)}


Guevara, F., Pinto, R., Rodríguez, L., Gómez, H., Ortiz, R., Ibrahim, M., Cruz, G. 2011. Precepciones locales de la degradación en una comunidad ganadera de Chiapas, México. Revista Cubana de Ciencia Agrícola. 45 (3): 311-319.

Loening, L. y Markussen, M. 2003. Pobreza, deforestación y sus eventuales implicaciones para la biodiversidad en Guatemala. Economia, Sociedad y Territorio. 4 (14): 279-315. https://doi.org/10.22136/est002003428

Martínez, I. y Cruz, M. 2009. El uso de químicos veterinarios y agrícolas en la zona ganadera de Xico, centro de Veracruz, México y el posible impacto ambiental. Acta Zoológica Mexicana. 25 (3) 673-681. https://doi.org/10.21829/azm.2009.253672

Moreno, P., Infante, D. y Madero, C. 2011. Germinación y supervivencia de dos especies de acahual de selva baja para restaurar dunas costeras. Revista mexicana de ciencias forestales. 2 (6): 19-36 https://doi.org/10.29298/rmcf.v2i6.572

Murgueitio, E. y Calle, Z. 1998. Diversidad biológica en sistemas de ganadería bovina en Colombia. In Conferencia electrónica de la FAO sobre Agroforestería para la producción animal en Latinoamérica (pp. 27-46). Roma: FAO. https://doi.org/10.3989/redc.2014.3.1133
Olivares, J., Avilés, F., Albarrán, B., Rojas, S. y Castelán, O. 2011. Identificación, uso y medición de leguminosas arbóreas en ranchos ganaderos del sur del estado de México. Tropical and Subtropical Agroecosystems. 14: 739-748.

Peralta-Pelaez, L. A. 2007. Diseño de un índice de integridad biótica para los lagos interdunarios de la región costera central del estado de Veracruz, México. Tesis de Doctorado. Instituto de Ecología A.C. Xalapa, Ver., México. 130 p. https://doi.org/10.1590/s1519-566x20070003000 03

Ramírez, C., Vera, G., Carrillo, F., Magaña, O. 2008. El cedro rojo (Cedrela odorata L.) como alternativa de reconversión en terrenos abandonados por la agricultura comercial en el sur de Tamaulipas. Agricultura Técnica en México. 34 (2): 243-250. https://doi.org/10.22231/asyd.v10i4.137

Zapata, A., Murgueitio, E., Mejía, C., Felipe, A. e Ibrahim, M. 2007. Efecto del pago por servicios ambientales en la adopción de sistema silvopastoriles en paisajes ganaderos de la cuenca media del río La Vieja, Colombia. Agroforesteria en las Américas. 45: 86-92. https://doi.org/10.32645/13906925.269

Copyright (c) 2014 Carlos Nahin Castro José, Ana Lid Del Angel Pérez y Mabel Hemández Osorio

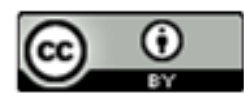

Este texto está protegido por una licencia Creative Commors 4.0 .

Usted es libre para Compartir —copiar y redistribuir el material en cualquier medio o formato- y Ad aptar el documento —remezclar, transformar y crear a partir del material- para cualquier propósito, inchso para fines comerciales, siempre que cumpla la condición de:

Atribución Usted debe dar crédito a la obra original de manera adecuada, proporcionar un enlace a la licencia, e indicar si se han realizado cambios. Puede hacerlo en cualquier forma razonable, pero no de forma tal que sugiera que tiene el apoyo del licenciante olo recibe por el usoque hace de la obra.

Resumendelicencia - Textocompletodelalicencia 